\title{
Identification and validation of HELLS (Helicase, Lymphoid- Specific) and ICAM1 (Intercellular adhesion molecule 1) as potential diagnostic biomarkers of lung cancer
}

\author{
Wei Zhu ${ }^{\text {Equal first author, } 1}$, Lin Lin Li ${ }^{\text {Equal first author, } 1}$, Yiyan Songyang ${ }^{1}$, Zhan Shi ${ }^{2}$, De Jia Li ${ }^{\text {Corresp. } 1}$ \\ ${ }^{1}$ Department of Occupational and Environmental Health, Wuhan University, School of Public Health, Wuhan, Hubei, China \\ 2 HUMAN BIOLOGY PROGRAM, University of Toronto, Toronto, Toronto, Canada \\ Corresponding Author: De Jia Li \\ Email address: djli@whu.edu.cn
}

Although lung cancer is one of the greatest threats to human health, its signaling pathway and related genes are still unknown. This study integrates data from three groups of people to study potential key candidate genes and pathways related to lung cancer. Expression profiles (GSE18842, GSE19188, and GSE27262), including 162 tumor tissue and 135 adjacent normal lung tissue samples, were integrated and analyzed. Differentially expressed genes (DEGs) and candidate genes were identified, their expression pathways were analyzed, and the diethylene glycol-related protein-protein interaction (PPI) network was analyzed. We identified 232 shared DEGs (40 upregulated and 192 down-regulated) from the three GSE datasets. The DEGs were clustered according to function and signaling pathway for significant enrichment analysis. In total, 129 nodes/DEGs were identified from the DEG PPI network complex. An improved prognosis was associated with increased HELLS (Helicase, Lymphoid-Specific) and decreased ICAM1 (Intercellular adhesion molecule 1) mRNA expression in lung cancer patients. In conclusion, we used integrated bioinformatics analysis to identify candidate genes and pathways in lung cancer to show that HELLS and ICAM1 might be the key genes related to tumorigenesis or tumor progression in lung cancer. Additional studies are needed to further explore the involved functional mechanisms. 
1 Identification and validation of HELLS (Helicase, Lymphoid-

2 Specific) and ICAM1 (Intercellular adhesion molecule 1) as

3 potential diagnostic biomarkers of lung cancer

4

5

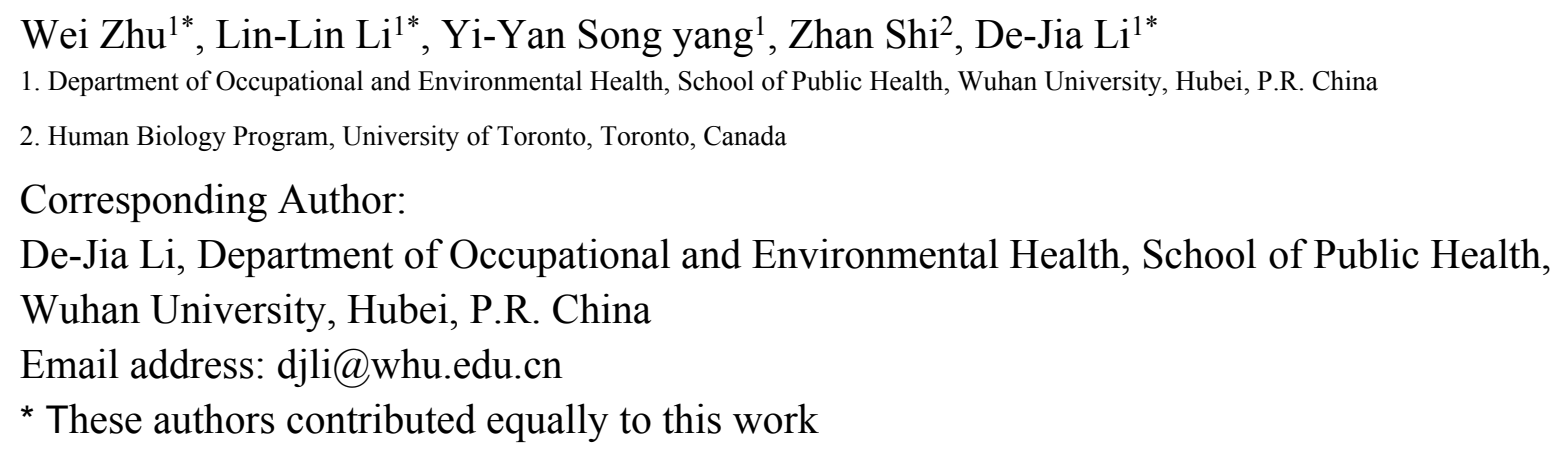

\section{Abstract}

Although lung cancer is one of the greatest threats to human health, its signaling pathway and related genes are still unknown. This study integrates data from three groups of people to study potential key candidate genes and pathways related to lung cancer. Expression profiles (GSE18842, GSE19188, and GSE27262), including 162 tumor tissue and 135 adjacent normal lung tissue samples, were integrated and analyzed. Differentially expressed genes (DEGs) and candidate genes were identified, their expression pathways were analyzed, and the diethylene glycol-related protein-protein interaction (PPI) network was analyzed. We identified 232 shared DEGs (40 upregulated and 192 down-regulated) from the three GSE datasets. The DEGs were clustered according to function and signaling pathway for significant enrichment analysis. In total, 129 nodes/DEGs were identified from the DEG PPI network complex. An improved prognosis was associated with increased HELLS (Helicase, Lymphoid-Specific) and decreased ICAM1 (Intercellular adhesion molecule 1) mRNA expression in lung cancer patients. In conclusion, we used integrated bioinformatics analysis to identify candidate genes and pathways in lung cancer to show that HELLS and ICAM1 might be the key genes related to tumorigenesis or tumor progression in lung cancer. Additional studies are needed to further explore the involved functional mechanisms.

\section{Introduction}

Lung cancer is one of the most leading causes of cancer-related deaths worldwide. In 2016, there were more than 220,000 diagnoses and nearly 158,000 deaths from lung cancer in the United States alone (Siegel et al., 2016). There are two main histological types of lung cancer: non-small cell lung cancer (NSCLC) and small cell lung cancer. The former accounts for about $85 \%$ of all lung cancer, including squamous cell carcinoma, adenocarcinoma and large cell carcinoma (Siegel et al., 2018). Although significant progress has been made in diagnosis and treatment methods in the last five years, the overall survival (OS) rate of lung cancer is still less than 15\% (Bironzo et al., 
2019). Therefore, the molecular mechanisms involved in the development of lung cancer should be studied and clarified in order to improve survival rates.

Gene chip, or gene expression profile, is a genetic detection technology that is particularly useful for screening differential gene expression since it has the ability to rapidly detect all genes expressed in the same sample after the time of sampling (Vogelstein et al., 2013). The widespread use of gene chips has generated a large amount of core slice data stored in public databases. Useful information has been provided from integrating and analyzing this data. In recent years, a large number of microarray data analyses on lung cancer have been conducted, and hundreds of differentially expressed genes have been identified. However, due to the heterogeneity of the tissue or samples in the existing studies, the results were limited or inconsistent. In cancer tissues, heterogeneity means that cells with different gene mutations may have different biological characteristics. The clinical diagnosis of cancer by pathologists usually relies on limited samples of cancer tissue that do not represent heterogeneity, either between or within patients (Bedard et al., 2013; de Sousa \& Carvalho, 2018).

As a result, reliable biomarkers have not been found in lung cancer. A novel approach to address these shortcomings is to incorporate a comprehensive bioinformatics approach to expression profiling techniques, which is the approach we adopted in this study.

We first downloaded three original microarray datasets, GSE18842 (Sanchez-Palencia et al., 2011a), GSE19188 (Hou et al., 2010a), and GSE27262 (Wei et al., 2014a; Wei et al., 2012a), from the NCBI Gene Expression Synthesis Database (NCBI-GEO, https://www.ncbi.nlm. nih. gov/geo) (Barrett et al., 2005). Data were obtained from 162 lung cancer cases and 135 adjacent normal tissues. The principles of our dataset selection were as following: 1 . the sample size was greater than $50 ; 2$. the samples were all from lung cancer patients and paracancer tissues; 3 . these patients had not undergone any other drug intervention; and 4. the purposes of carrying out the gene chip or RNA-seq were to compare and analyze the RNA expression differences between lung cancer patients and paracancer tissues. We screened the corresponding DEGs according to the data processing standards of the Morpheus website and used DAVID, Cytoscape, Metascape (http://metascape. org/) (Zhou et al., 2019), UCSC (https://genomecancer.ucsc.edu/) (Haeussler et al., 2019), cBioportal (Gao et al., 2013), BioCyc(http://biocyc.org) (Latendresse et al., 2012), and Panther (http://www.pantherdb.org) to perform gene ontology and pathway enrichment analysis. We also developed a comprehensive DEG protein-protein interaction (PPI) network and module analysis to identify the central gene of lung cancer using the Search Tool for the Retrieval of Interacting Genes/Proteins database (STRING, http://string db.org). To identify the central lung cancer genes using string (http://string db.org), we also developed a comprehensive DEG protein-protein interaction (PPI) network and module analysis. HELLS and ICAM1 were identified, and their biological functions and key pathways were enriched to ascertain more accurate and practical biomarkers for the early diagnosis, individualized prevention, and treatment of lung cancer. Finally, we analyzed the expression of HELLS and ICAM1 in lung cancer patients to determine their expression patterns, potential functions, and different prognostic values.

\section{Materials \& Methods}


83

84

85

86

87

88

89

90

91

92

93

94

95

96

97

98

99

100

101

102

103

104

105

106

107

108

109

110

111

112

113

114

115

116

117

118

119

120

121

\section{Microarray data analysis and identification of DEGs}

We obtained lung cancer and adjacent tissue gene expression profiles for GSE18842, GSE19188, and GSE27262 from NCBI-GEO, which is a free microarray/gene database repository of high throughput gene expression data. Microarray data for GSE18842 were based on the GPL570 platform [(HG-U133_Plus_2) Affymetrix Human Genome U133 Plus 2.0 Array], and included 46 tumors and 45 controls (submission date: November 2, 2009) (Latendresse et al., 2012). GSE19188 data were based on the GPL570 platform [(HG-U133_Plus_2) Affymetrix Human Genome U133 Plus 2.0 Array], and included 91 tumor and 65 adjacent normal lung tissue samples (submission date: November 25, 2009) (Hou et al., 2010b). GSE27262 data were based on the GPL570 platform ([HG-U133_Plus_2] Affymetrix Human Genome U133 Plus 2.0 Array), and included 25 pairs of tumor and adjacent normal tissues from lung adenocarcinoma patients (submission date: February 11, 2011) (Wei et al., 2014b; Wei et al., 2012b). We selected these three datasets for integrated analysis and identified DEGs using a classical $t$ test. The adjusted $\mathrm{p}$ values (adj. $\mathrm{p}$ ) were utilized to correct the occurrence of false-positive results using the Benjamini and Hochberg false discovery rate method by default. In the present study, statistically significant DEGs were defined using values of adj.p $<0.05$ and $[\log \mathrm{FC}]>1$ as cutoff criteria (2015; Ashburner et al., 2000; Lebrec et al., 2009).

\section{Gene ontology (GO) and pathway enrichment analysis}

Metascape (http://metascape.org/) is an online analysis tool for extracting comprehensive biometric information from huge lists of candidate genes. It not only performs typical genetic terminology enrichment analysis, but also visualizes the relationship between genomic terms, searches for interesting and related genes or terms, and dynamically views genes from their biological functions and pathways. GO analysis and the Kyoto Genomics and Genomics Encyclopedia (KEGG) path analysis were conducted on the selected DEG using the Metascape tool. The enrichment score [- $\log _{10}$ (p-value)] was significantly ranked with a $p$-value $<0.01$ as the cutoff criterion.

\section{Integration of PPI network complex identification}

We developed a DEG-encoded protein and PPI network using STRING (http://string-db.org) (Szklarczyk et al., 2019). The PPI network was constructed using Cytoscape software (version 3.7.1) to analyze the interactions between candidate DEG-encoded proteins in lung cancer (Kohl et al., 2011). The Node Analyzer was calculated using the Network Analyzer plug-in, which reveals the number of connections used to filter the PPI hub genes. The corresponding protein identified at the central node may be a core protein and key candidate gene with important physiological regulatory functions.

\section{Identification and clinical significance of central genes}

Hub genes were identified using CythopCAE's CyoHubBA toolkit. The top 10 central genes with less than 10 degrees were selected. Hierarchical clustering of hub genes in the Cancer Genome Atlas (TCGA) database was constructed using the UCSC Cancer Genome Browser (https://genome cancer.ucsc.edu/). Biological process analysis of the hub gene was performed 
122 utilizing the Cytoscape Bionetwork Gene Oncology Tool (BiNGO) plug-in. The frequency of gene 123 changes was assessed using the cBioportal online database (http://www.cbioportal.org/). PPI 124 networks were built using STRING.

\section{Prognosis analysis using Kaplan-Meier plots}

126

127

128

129

130

131

132

133

134

135

136

137

138

139

140

141

142

143

144

145

146

147

148

149

150

151

152

153

154

155

156

157

158

The TCGA online database, which contains gene expression data and survival information for lung cancer patients and sequencing and pathological data for 30 different cancers, was utilized to evaluate the prognostic value of DEG expression. To analyze the OS of patients with lung cancer, 545 patients were assigned to either two groups (high and low expression) or three groups (high, medium, and low expression) by median expression and were assessed using Kaplan-Meier survival analysis with hazard ratio, $95 \%$ confidence intervals, and log-rank p-value. Only the JetSet best probe set of DEGs was selected to obtain a Kaplan-Meier plot, with the risk number shown below the main plot.

\section{Lung cancer samples}

Lung cancer and adjacent normal tissues were obtained after surgical resection of patients with NSCLC being treated at Wuhan University Affiliated Hospital, Hubei Provincial People's Hospital, and Zhongnan Hospital of Wuhan University, China. Informed consent was received by each patient. The study was approved by Wuhan University's Institute of Ethics with certificate number 2018001. It is presumed that informed consent had been obtained for all datasets used from the published literature.

\section{RT-qPCR}

Total RNA was extracted from lung cancer cells and tissues using Trizol reagent (Invitrogen; Thermo Fisher Scientific, Inc., USA). A reverse transcription kit (Vazyme Biotech Co., China) was used to reverse transcribe RNA and mRNA expression was evaluated using the $2^{\wedge}(\Delta \Delta \mathrm{ct})$ method. Relative gene expression was then calculated and normalized to endogenous glyceraldehyde 3-phosphate dehydrogenase (GAPDH). The primers of GAPDH, HELLS, ICAM1 were purchased from Qingke Biotechnology Co., Ltd., China. The forward and reverse primer sequences are as follows: GAPDH F :5'-CCTTCCGTGTCCCCACT-3' and GAPDH R:5'GCCTGCTTCACCACCTTC-3', HELLS F: 5'-CCCTCCTTTCTTCTAGTAATGCAGTT-3' and HELLS R: 5'-CCCAATCTCTCCCCATGAAAA-3'; ICAM1 F:5'GAACCCATTGCCCGAGCTCA-3' and ICAM1 R: 5'-TGACAGTCACTGATTCCCCGAT-3'.

\section{Cell culture}

Human NSCLC cell lines A549, H1299, PC9, and HCC827 and the normal lung epithelial cell line BEAS2B were all bought from the Shanghai Cell Bank of the Chinese Academy of Sciences and were cryopreserved in liquid nitrogen tanks. The cells were cultured in DubCo's MudieEdEdE medium (Hyo Corporation, USA) with 10\% fetal bovine serum (GiBCO Co., USA) and incubated at $37^{\circ} \mathrm{C}$ in a $5 \% \mathrm{CO} 2$ atmosphere.

\section{Statistical analysis}


159 Experimental data were recorded in Excel and analyzed using GraphPad Prism 7. The results were

160

161

162

163

164

165

166

167

168

169

170

171

172

173

174

175

176

177

178

179

180

181

182

183

184

185

186

187

188

189

190

191

192

193

194

195

196

analyzed by a two-tailed $t$ test. Values of $\mathrm{p}<0.05$ were considered significant differences. Data were expressed as mean \pm standard error.

\section{Results}

\section{Identification of DEGs in lung cancer}

Lung cancer and adjacent normal tissue gene expression profiles for GSE18842, GSE19188, and GSE27262 were obtained from NCBI-GEO. Microarray data for GSE18842 comprised 46 tumors and 45 controls (Sanchez-Palencia et al., 2011b). GSE21815 data comprised 91 tumor and 65 adjacent normal lung tissue samples. GSE27262 data included 25 paired tumor and adjacent normal tissue samples. In total, 2042, 1424, and 1142 DEGs were extracted from the GSE18842, GSE19188, and GSE27262 expression profile datasets, respectively, using adj.p $<0.05$ and $[\log \mathrm{FC}]>1$ as cutoff criteria. A total of 232 uniformly expressed genes were identified from the three profile datasets using integrated analysis (Fig. 1-2). When compared to normal lung tissue, lung cancer tissues included 40 up-regulated genes and 192 down-regulated genes (Table 1).

\section{Functional and pathway enrichment analysis of DEGs}

The functions and pathways of the candidate DEGs were predicted using the Metascape Database (Table 2) (Geiman et al., 1998a). There were 18 terms and two pathways involved in the DEGs enrichment analysis (Fig. 1A), and the DEGs were mainly enriched during developmental growth, embryonic morphogenesis, cell-substrate junction assembly, renin secretion, regulation of cell adhesion, myeloid leukocyte activation, mesenchyme development, assembly of cellular components involved in morphogenesis and muscle system processes, in the cell surface receptor signaling pathway involved in cell-cell signaling, positive regulation of protein transport, endodermal cell differentiation, negative regulation of cell proliferation, hemostasis, and ameboidal-type cell migration. The GO function and KEGG pathway enrichment analysis of candidate DEGs are shown in Figure 1. The enriched terms were closely connected with each other and clustered into intact networks (Fig. 1B). These results indicate that most DEGs are significantly enriched during cardiomyocyte proliferation, protein binding, and the positive regulation of cell membranes.

\section{DEGs modular analysis with PPI network and hub gene identification}

Protein interaction networks have proven to be powerful tools for predicting new essential genes in specific signal transduction pathways. Using the STRING online database and Cytoscape software 13, a total of 232 DEGs were filtered into the PPI network complex. The PPI network of DEGs was constructed (Fig. 2B with the most important module obtained using the Cytoscape (Fig. 2C). Using the Metascope to analyze the functions of the genes involved in this module, we found that the functions mainly focused on cell division and pre-mitotic stage and mitosis cell cycle transition. (Fig. 3).

\section{Hub gene selection and analysis}


197 The top 10 node degree genes were MAD2L1, POLQ, HELLS, ANLN, BIRC5, ATAD2, CCNB2,

198

199

200

201

202

203

204

205

206

207

208

209

210

211

212

213

214

215

216

217

218

219

220

221

222

223

224

225

226

227

228

229

230

231

232

233

234

PTK2, ICAM1, and ITGAX (Table 3). A network of hub genes and their co-expressed genes were analyzed using the cBioPortal online platform (Fig. 4A). The analysis of the biological processes of the hub genes, which was constructed using plug-in BiNGO, is shown in Fig. 4C. Hierarchical clustering indicated that the hub genes could differentiate cancer samples from noncancerous samples (Fig. 4B). These genes may play a significant role in lung cancer development.

\section{Association between HELLS and ICAM1 expression and prognoses in lung} cancer patients

We used the TCGA website to further explore the 10 central genes related to survival of lung cancer patients. According to curve and logarithmic rank test analysis, the elevated level of HELLS mRNA correlated significantly with OS difference in lung cancer patients (Fig. 5). Interestingly, lower ICAM1 levels also indicated poor prognoses in lung cancer patients (Fig. 6). After assessing the mRNA levels of ICAM1 and HELLS using the Oncomine online database (https://www. oncomine.org/resource/login.html ) (Rhodes et al., 2004) (Fig. 7A, B), it was indicated that ICAM1 was down-regulated in lung cancer across five differet studies. Furthermore, HELLS expression was upregulated in lung cancer tumors. After HELLS and ICAM1 were identified from these 10 central genes, Gene Expression Profiling interactive analysis (GEPIA; http://gepia.cancer-pku.cn/) was used to validate the selected upregulated and downregulated genes (Tang et al., 2017). The GEPIA analysis includes data from the Cancer Genome Atlas (TCGA) and the Genotype Tissue Expression (GTEx), and provides online gene expression level analysis, survival analysis, and tumor staging analysis for 33 types of cancers, including lung adenocarcinoma (LUAD) and lung squamous cell carcinoma (LUSC). The mRNA level of HELLS was evaluated in lung cancer using GEPIA analysis, and the expression of HELLS in lung cancer tissues was significantly higher than in adjacent tissues $(p<0.05)$ (Fig. 5G). The expression of ICAM-1 in LUSC was significantly lower than in paracancer tissues $(p<0.05)$ (Fig. 6G).

A PPI network of ICAM1 and HELLS was constructed using the STRING database. The results indicated that HELLS was associated with other genes in the minichromosome maintenance protein (MCMp) family, such as MCM5, MCM3, and MCM2 (Fig. 7C)._Interactions between ICAM1 and other genes associated with inflammation were also observed in the present study (Fig. 7D). To further assess the expression of ICAM1 and HELLS, we measured mRNA levels in 79 cases of lung cancer and paired paracancer samples. RT-qPCR results showed that HELLS expression in lung cancer tissues was upregulated when compared with normal tissue, while ICAM1 expression in lung cancer tissues was downregulated when compared with normal tissue. When compared with the normal lung cancer cell line BEAS2B, HELLS mRNA levels in human lung cancer cell lines H1299, A549 and HCC827 were remarkably highly expressed. However, the expression of ICAM1 only decreased in HCC 827 when compared with the normal lung epithelial cell line BEAS2B (Fig. 8). 


\section{Discussion}

236 Over the past few decades, experts have explored the causes and underlying mechanisms of lung 237 cancer formation and development through extensive basic and clinical research. However, most

238

239

240

241

242

243

244

245

246

247

248

249

250

251

252

253

254

255

256

257

258

259

260

261

262

263

264

265

266

267

268

269

270

271

272

273

274 previous studies focused on the results of a single genetic event or single cohort study with inconsistent and incomplete results, and the incidence and mortality rate of lung cancer worldwide remain high (Zeng et al., 2019; Ning et al., 2019). Our study integrated three cohort profile datasets from different studies and used bioinformatics methods to perform an in-depth analysis, identifying 232 frequent changes in DEGS (40 up-regulations and 192 down-regulations). The 232 DEGs were then allocated to three groups according to GO terminology (molecular function, biological process, and cell component) using a variety of methods. GO and KEGG analyses showed significant enrichment in these DEGs based on function and signaling pathway analysis. A PPI network complex was developed for the DEGs to filter the hub genes and dysregulated pathways. To determine the expression pattern, potential function, and different prognostic values of the DEGS, we performed a detailed analysis of the expression of DEGS in lung cancer patients.

HELLS (also known as SMARCA6 and PASG) is the main member of the SNF2 chromatin remodeling enzyme family. The human HELLS gene is located in the c3-d1 region of chromosome 10q23-q24, while the mouse homolog is located in the same region of chromosome 19. Related studies have shown that HELLS plays an important regulatory role during normal embryonic development (Sun et al., 2004) and encodes a lymphoid-specific helicase. Other helicases are involved in processes involving DNA strand separation, including replication, repair, recombination, and transcription. Lymphoid-specific helicase has been shown to be involved with cellular proliferation (Geiman et al., 1998b; Lee et al., 2000). To maintain the DNA methylation pattern of the mammalian genome (Myant \& Stancheva, 2008), HELLS typically interacts with DNA methyltransferases. According to recent research, HELLS also plays an important regulatory role in cell proliferation and, possibly, in the development of cancer (He et al., 2016; Benavente et al., 2014; Teh et al., 2012; Tao et al., 2011). HELLS is a key epigenetic driver of hepatocellular carcinoma (HCC) and inhibits multiple tumor suppressor genes by promoting the occupancy of nucleosomes of NFR and enhancers to promote HCC progression (Law et al., 2019). Recent studies have shown that HELLS genes are upregulated in nasopharyngeal carcinoma, retinoblastoma, head and neck cancer, and breast cancer. However, the detailed mechanisms of HELLS in cancer, particularly the reasons for its differential expression and downstream targets, need further research. The present study screened several DEGs in three datasets to reveal that increased levels of HELLS mRNA were significantly associated with poor OS in lung cancer patients, suggesting that HELLS may be a potential novel predictor of prognosis.

ICAM1 (Intercellular adhesion molecule 1) is an important member of the immunoglobulin superfamily. It is a glycosylated transmembrane protein that plays a key role in immune synapse formation, T cell activation, leukocyte trafficking, and various cellular immune responses. A large number of studies have shown that ICAM1 shows higher expression in mesenchymal stem cells such as bone marrow, placenta, fat, and periodontal ligament (Brooke et al., 2008; De Francesco et al., 2009; Sununliganon \& Singhatanadgit, 2012). Studies have also shown that ICAM-1 is a 
275 marker of human and mouse liver cancer stem cells and is involved in the metastasis of liver cancer 276 cells. Its expression is regulated by the stem cell transcription factor Nanog (Liu et al., 2013). 277 Reduced expression of ICAM-1 could play a role in the suppression of tumor progression in many 278 cancer cells, such as breast cancer (Ogawa et al., 1998), gastric cancer (Fujihara et al., 1999), lung 279 cancer (Kotteas et al., 2014), and colorectal cancer (Maeda et al., 2002). Additionally, ICAM1 and 280 CD44 may have compensatory effects to maintain the dry characteristics of esophageal squamous 281 cell carcinoma, indicating multiple targeted therapies that can be combined and considered in 282 cancer treatment (Tsai et al., 2015). Research has demonstrated that ICAM1 is involved in 283 angiogenesis through the regulation of endothelial cell migration (Kevil et al., 2004). Additional 284 studies have shown that ICAM-1 in the systemic circulation of lung cancer patients can bind to 285 leukocyte-function associated antigen-1 (LFA-1) of cytotoxic lymphocytes in the blood, enabling cancer cells to evade immune recognition mechanisms (Kim et al., 2017). Other studies have shown that cannabinoid-induced ICAM-1 can increase LAK cell-mediated tumor cell killing ability in lung cancer, a novel antitumor mechanism of cannabinoids (Haustein et al., 2014). From these three datasets, we identified that ICAM-1 is dysregulated in lung cancer. In combination with a series of previous studies, we found that decreased ICAM1 mRNA levels predict poor prognoses in patients with lung cancer (Melis et al., 1996; Haustein et al., 2014; Schellhorn et al., 2015). Therefore, ICAM1 may be a novel potential therapy target for lung cancer patients.

295

296

297

298

299

300

301

302

303

304

305

306

307

308

309

310

311

312

313

314

315

316

\section{Conclusions}

In summary, we analyzed multiple cohort datasets and integrated bioinformatics to identify and screen 232 candidate genes, and we constructed a PIP network complex to screen 129 gene nodes and 10 node degree genes in DEGs. We found that elevated HELLS and decreased ICAM1 mRNA levels are predictive of poor prognoses in lung cancer patients, which could significantly improve our understanding of the causes and potential molecular events of lung cancer. However, our findings should be supplemented, and the direction for further research may include related mechanism validation studies. Whether the selected molecules have clinical significance should be verified and discussed. Therefore, further research is required to clarify the exact molecular mechanisms of these genes in lung cancer.

\section{References}

2012. Comprehensive molecular portraits of human breast tumours. NATURE 490:61-70. 10.1038/nature11412

2015. Gene Ontology Consortium: going forward. NUCLEIC ACIDS RESEARCH 43:D1049-D1056. 10.1093/nar/gku1179

Ashburner, M., Ball, C.A., Blake, J.A., Botstein, D., Butler, H., Cherry, J.M., Davis, A.P., Dolinski, K., Dwight, S.S., Eppig, J.T., Harris, M.A., Hill, D.P., Issel-Tarver, L., Kasarskis, A., Lewis, S., Matese, J.C., Richardson, J.E., Ringwald, M., Rubin, G.M., and Sherlock, G. 2000. Gene ontology: tool for the unification of biology. The Gene Ontology Consortium. NATURE GENETICS 25:25-29. 10.1038/75556

Barrett, T., Suzek, T.O., Troup, D.B., Wilhite, S.E., Ngau, W.C., Ledoux, P., Rudnev, D., Lash, A.E., Fujibuchi, W., 
317

318

319

320

321

322

323

324

325

326

327

328

329

330

331

332

333

334

335

336

337

338

339

340

341

342

343

344

345

346

347

348

349

350

351

352

353

354

355

356

357

358

359

360

361

362

363

364

365

366

367

368

369

370

371

372

and Edgar, R. 2005. NCBI GEO: mining millions of expression profiles--database and tools. NUCLEIC ACIDS RESEARCH 33:D562-D566. 10.1093/nar/gki022

Bedard, P.L., Hansen, A.R., Ratain, M.J., and Siu, L.L. 2013. Tumour heterogeneity in the clinic. NATURE 501:355364. 10.1038/nature12627

Benavente, C.A., Finkelstein, D., Johnson, D.A., Marine, J.C., Ashery-Padan, R., and Dyer, M.A. 2014. Chromatin remodelers HELLS and UHRF1 mediate the epigenetic deregulation of genes that drive retinoblastoma tumor progression. Oncotarget 5:9594-9608. 10.18632/oncotarget.2468

Bironzo, P., Passiglia, F., and Novello, S. 2019. Five-year overall survival of pembrolizumab in advanced non-small cell lung cancer: another step from care to cure? Ann Transl Med 7:S212. 10.21037/atm.2019.08.91

Brooke, G., Tong, H., Levesque, J.P., and Atkinson, K. 2008. Molecular trafficking mechanisms of multipotent mesenchymal stem cells derived from human bone marrow and placenta. STEM CELLS AND DEVELOPMENT 17:929-940. 10.1089/scd.2007.0156

De Francesco, F., Tirino, V., Desiderio, V., Ferraro, G., D'Andrea, F., Giuliano, M., Libondi, G., Pirozzi, G., De Rosa, A., and Papaccio, G. 2009. Human CD34/CD90 ASCs are capable of growing as sphere clusters, producing high levels of VEGF and forming capillaries. PLoS One 4:e6537. 10.1371/journal.pone.0006537

de Sousa, V., and Carvalho, L. 2018. Heterogeneity in Lung Cancer. PATHOBIOLOGY 85:96-107. $10.1159 / 000487440$

Fujihara, T., Yashiro, M., Inoue, T., Sawada, T., Kato, Y., Ohira, M., Nishiguchi, Y., Ishikawa, T., Sowa, M., and Chung, K.H. 1999. Decrease in ICAM-1 expression on gastric cancer cells is correlated with lymph node metastasis. Gastric Cancer 2:221-225. 10.1007/s101209900039

Gao, J., Aksoy, B.A., Dogrusoz, U., Dresdner, G., Gross, B., Sumer, S.O., Sun, Y., Jacobsen, A., Sinha, R., Larsson, E., Cerami, E., Sander, C., and Schultz, N. 2013. Integrative analysis of complex cancer genomics and clinical profiles using the cBioPortal. Science Signaling 6:11. 10.1126/scisignal.2004088

Geiman, T.M., Durum, S.K., and Muegge, K. 1998a. Characterization of gene expression, genomic structure, and chromosomal localization of Hells (Lsh). GENOMICS 54:477-483. 10.1006/geno.1998.5557

Geiman, T.M., Durum, S.K., and Muegge, K. 1998b. Characterization of gene expression, genomic structure, and chromosomal localization of Hells (Lsh). GENOMICS 54:477-483. 10.1006/geno.1998.5557

Haeussler, M., Zweig, A.S., Tyner, C., Speir, M.L., Rosenbloom, K.R., Raney, B.J., Lee, C.M., Lee, B.T., Hinrichs, A.S., Gonzalez, J.N., Gibson, D., Diekhans, M., Clawson, H., Casper, J., Barber, G.P., Haussler, D., Kuhn, R.M., and Kent, W.J. 2019. The UCSC Genome Browser database: 2019 update. NUCLEIC ACIDS RESEARCH 47:D853-D858. 10.1093/nar/gky1095

Haustein, M., Ramer, R., Linnebacher, M., Manda, K., and Hinz, B. 2014. Cannabinoids increase lung cancer cell lysis by lymphokine-activated killer cells via upregulation of ICAM-1. BIOCHEMICAL PHARMACOLOGY 92:312325. 10.1016/j.bcp.2014.07.014

He, X., Yan, B., Liu, S., Jia, J., Lai, W., Xin, X., Tang, C.E., Luo, D., Tan, T., Jiang, Y., Shi, Y., Liu, Y., Xiao, D., Chen, L., Liu, S., Mao, C., Yin, G., Cheng, Y., Fan, J., Cao, Y., Muegge, K., and Tao, Y. 2016. Chromatin Remodeling Factor LSH Drives Cancer Progression by Suppressing the Activity of Fumarate Hydratase. CANCER RESEARCH 76:5743-5755. 10.1158/0008-5472.CAN-16-0268

Hou, J., Aerts, J., den Hamer, B., van Ijcken, W., den Bakker, M., Riegman, P., van der Leest, C., van der Spek, P., Foekens, J.A., Hoogsteden, H.C., Grosveld, F., and Philipsen, S. 2010a. Gene expression-based classification of nonsmall cell lung carcinomas and survival prediction. PLoS One 5:e10312. 10.1371/journal.pone.0010312

Hou, J., Aerts, J., den Hamer, B., van Ijcken, W., den Bakker, M., Riegman, P., van der Leest, C., van der Spek, P., Foekens, J.A., Hoogsteden, H.C., Grosveld, F., and Philipsen, S. 2010b. Gene expression-based classification of nonsmall cell lung carcinomas and survival prediction. PLoS One 5:e10312. 10.1371/journal.pone.0010312

Kevil, C.G., Orr, A.W., Langston, W., Mickett, K., Murphy-Ullrich, J., Patel, R.P., Kucik, D.F., and Bullard, D.C. 2004. Intercellular adhesion molecule-1 (ICAM-1) regulates endothelial cell motility through a nitric oxide-dependent pathway. JOURNAL OF BIOLOGICAL CHEMISTRY 279:19230-19238. 10.1074/jbc.M312025200

Kim, E., Kim, W., Lee, S., Chun, J., Kang, J., Park, G., Han, I., Yang, H.J., Youn, H., and Youn, B. 2017. TRAF4 promotes lung cancer aggressiveness by modulating tumor microenvironment in normal fibroblasts. Sci Rep 7:8923. 10.1038/s41598-017-09447-z

Kohl, M., Wiese, S., and Warscheid, B. 2011. Cytoscape: software for visualization and analysis of biological networks. Methods Mol Biol 696:291-303. 10.1007/978-1-60761-987-1_18

Kotteas, E.A., Boulas, P., Gkiozos, I., Tsagkouli, S., Tsoukalas, G., and Syrigos, K.N. 2014. The intercellular cell adhesion molecule-1 (icam-1) in lung cancer: implications for disease progression and prognosis. ANTICANCER RESEARCH 34:4665-4672.

Latendresse, M., Paley, S., and Karp, P.D. 2012. Browsing metabolic and regulatory networks with BioCyc. Methods

Peer) reviewing PDF | (2019:10:42669:1:1:NEW 10 Feb 2020) 
Mol Biol 804:197-216. 10.1007/978-1-61779-361-5_11

Law, C.T., Wei, L., Tsang, F.H., Chan, C.Y., Xu, I.M., Lai, R.K., Ho, D.W., Lee, J.M., Wong, C.C., Ng, I.O., and Wong, C.M. 2019. HELLS Regulates Chromatin Remodeling and Epigenetic Silencing of Multiple Tumor Suppressor Genes in Human Hepatocellular Carcinoma. HEPATOLOGY 69:2013-2030. 10.1002/hep.30414

Lebrec, J.J., Huizinga, T.W., Toes, R.E., Houwing-Duistermaat, J.J., and van Houwelingen, H.C. 2009. Integration of gene ontology pathways with North American Rheumatoid Arthritis Consortium genome-wide association data via linear modeling. BMC Proc 3 Suppl 7:S94. 10.1186/1753-6561-3-s7-s94

Lee, D.W., Zhang, K., Ning, Z.Q., Raabe, E.H., Tintner, S., Wieland, R., Wilkins, B.J., Kim, J.M., Blough, R.I., and Arceci, R.J. 2000. Proliferation-associated SNF2-like gene (PASG): a SNF2 family member altered in leukemia. CANCER RESEARCH 60:3612-3622.

Liu, S., Li, N., Yu, X., Xiao, X., Cheng, K., Hu, J., Wang, J., Zhang, D., Cheng, S., and Liu, S. 2013. Expression of intercellular adhesion molecule 1 by hepatocellular carcinoma stem cells and circulating tumor cells. GASTROENTEROLOGY 144:1031-1041. 10.1053/j.gastro.2013.01.046

Maeda, K., Kang, S.M., Sawada, T., Nishiguchi, Y., Yashiro, M., Ogawa, Y., Ohira, M., Ishikawa, T., and HirakawaYS, C.K. 2002. Expression of intercellular adhesion molecule-1 and prognosis in colorectal cancer. $O N C O L O G Y$ REPORTS 9:511-514.

Melis, M., Spatafora, M., Melodia, A., Pace, E., Gjomarkaj, M., Merendino, A.M., and Bonsignore, G. 1996. ICAM-1 expression by lung cancer cell lines: effects of upregulation by cytokines on the interaction with LAK cells. EUROPEAN RESPIRATORY JOURNAL 9:1831-1838. 10.1183/09031936.96.09091831

Myant, K., and Stancheva, I. 2008. LSH cooperates with DNA methyltransferases to repress transcription. MOLECULAR AND CELLULAR BIOLOGY 28:215-226. 10.1128/MCB.01073-07

Ning, Y., Liu, W., Guan, X., Xie, X., and Zhang, Y. 2019. CPSF3 is a promising prognostic biomarker and predicts recurrence of non-small cell lung cancer. Oncology Letters 18:2835-2844. 10.3892/ol.2019.10659

Ogawa, Y., Hirakawa, K., Nakata, B., Fujihara, T., Sawada, T., Kato, Y., Yoshikawa, K., and Sowa, M. 1998. Expression of intercellular adhesion molecule-1 in invasive breast cancer reflects low growth potential, negative lymph node involvement, and good prognosis. CLINICAL CANCER RESEARCH 4:31-36.

Rhodes, D.R., Yu, J., Shanker, K., Deshpande, N., Varambally, R., Ghosh, D., Barrette, T., Pandey, A., and Chinnaiyan, A.M. 2004. ONCOMINE: a cancer microarray database and integrated data-mining platform. NEOPLASIA 6:1-6. 10.1016/s1476-5586(04)80047-2

Sanchez-Palencia, A., Gomez-Morales, M., Gomez-Capilla, J.A., Pedraza, V., Boyero, L., Rosell, R., and Farez-Vidal, M.E. 2011a. Gene expression profiling reveals novel biomarkers in nonsmall cell lung cancer. INTERNATIONAL JOURNAL OF CANCER 129:355-364. 10.1002/ijc.25704

Sanchez-Palencia, A., Gomez-Morales, M., Gomez-Capilla, J.A., Pedraza, V., Boyero, L., Rosell, R., and Farez-Vidal, M.E. 2011b. Gene expression profiling reveals novel biomarkers in nonsmall cell lung cancer. INTERNATIONAL JOURNAL OF CANCER 129:355-364. 10.1002/ijc.25704

Schellhorn, M., Haustein, M., Frank, M., Linnebacher, M., and Hinz, B. 2015. Celecoxib increases lung cancer cell lysis by lymphokine-activated killer cells via upregulation of ICAM-1. Oncotarget 6:39342-39356. 10.18632/oncotarget.5745

Siegel, R.L., Miller, K.D., and Jemal, A. 2016. Cancer statistics, 2016. CA Cancer J Clin 66:7-30. 10.3322/caac.21332 Siegel, R.L., Miller, K.D., and Jemal, A. 2018. Cancer statistics, 2018. CA Cancer J Clin 68:7-30. 10.3322/caac.21442 Sun, L.Q., Lee, D.W., Zhang, Q., Xiao, W., Raabe, E.H., Meeker, A., Miao, D., Huso, D.L., and Arceci, R.J. 2004. Growth retardation and premature aging phenotypes in mice with disruption of the SNF2-like gene, PASG. Genes Dev 18:1035-1046. 10.1101/gad.1176104

Sununliganon, L., and Singhatanadgit, W. 2012. Highly osteogenic PDL stem cell clones specifically express elevated levels of ICAM1, ITGB1 and TERT. CYTOTECHNOLOGY 64:53-63. 10.1007/s10616-011-9390-5

Szklarczyk, D., Gable, A.L., Lyon, D., Junge, A., Wyder, S., Huerta-Cepas, J., Simonovic, M., Doncheva, N.T., Morris, J.H., Bork, P., Jensen, L.J., and Mering, C.V. 2019. STRING v11: protein-protein association networks with increased coverage, supporting functional discovery in genome-wide experimental datasets. NUCLEIC ACIDS RESEARCH 47:D607-D613. 10.1093/nar/gky1131

Tang, Z., Li, C., Kang, B., Gao, G., Li, C., and Zhang, Z. 2017. GEPIA: a web server for cancer and normal gene expression profiling and interactive analyses. NUCLEIC ACIDS RESEARCH 45:W98-W102. 10.1093/nar/gkx247 Tao, Y., Liu, S., Briones, V., Geiman, T.M., and Muegge, K. 2011. Treatment of breast cancer cells with DNA demethylating agents leads to a release of Pol II stalling at genes with DNA-hypermethylated regions upstream of TSS. NUCLEIC ACIDS RESEARCH 39:9508-9520. 10.1093/nar/gkr611

Teh, M.T., Gemenetzidis, E., Patel, D., Tariq, R., Nadir, A., Bahta, A.W., Waseem, A., and Hutchison, I.L. 2012. FOXM1 induces a global methylation signature that mimics the cancer epigenome in head and neck squamous cell 
429

430

431

432

433

434

435

436

437

438

439

440

441

442

443

444

445

446

447

448

449

450

451

452

453

454

455

carcinoma. PLoS One 7:e34329. 10.1371/journal.pone.0034329

Tsai, S.T., Wang, P.J., Liou, N.J., Lin, P.S., Chen, C.H., and Chang, W.C. 2015. ICAM1 Is a Potential Cancer Stem Cell Marker of Esophageal Squamous Cell Carcinoma. PLoS One 10:e142834. 10.1371/journal.pone.0142834

Vogelstein, B., Papadopoulos, N., Velculescu, V.E., Zhou, S., Diaz, L.J., and Kinzler, K.W. 2013. Cancer genome landscapes. SCIENCE 339:1546-1558. 10.1126/science.1235122

Wei, T.Y., Hsia, J.Y., Chiu, S.C., Su, L.J., Juan, C.C., Lee, Y.C., Chen, J.M., Chou, H.Y., Huang, J.Y., Huang, H.M., and $\mathrm{Yu}, \mathrm{C} . \mathrm{T}$. 2014a. Methylosome protein 50 promotes androgen- and estrogen-independent tumorigenesis. CELLULAR SIGNALLING 26:2940-2950. 10.1016/j.cellsig.2014.09.014

Wei, T.Y., Hsia, J.Y., Chiu, S.C., Su, L.J., Juan, C.C., Lee, Y.C., Chen, J.M., Chou, H.Y., Huang, J.Y., Huang, H.M., and $\mathrm{Yu}, \mathrm{C} . \mathrm{T}$. 2014b. Methylosome protein 50 promotes androgen- and estrogen-independent tumorigenesis. CELLULAR SIGNALLING 26:2940-2950. 10.1016/j.cellsig.2014.09.014

Wei, T.Y., Juan, C.C., Hisa, J.Y., Su, L.J., Lee, Y.C., Chou, H.Y., Chen, J.M., Wu, Y.C., Chiu, S.C., Hsu, C.P., Liu, K.L., and Yu, C.T. 2012a. Protein arginine methyltransferase 5 is a potential oncoprotein that upregulates G1 cyclins/cyclin-dependent kinases and the phosphoinositide 3-kinase/AKT signaling cascade. CANCER SCIENCE 103:1640-1650. 10.1111/j.1349-7006.2012.02367.x

Wei, T.Y., Juan, C.C., Hisa, J.Y., Su, L.J., Lee, Y.C., Chou, H.Y., Chen, J.M., Wu, Y.C., Chiu, S.C., Hsu, C.P., Liu, K.L., and Yu, C.T. 2012b. Protein arginine methyltransferase 5 is a potential oncoprotein that upregulates G1 cyclins/cyclin-dependent kinases and the phosphoinositide 3-kinase/AKT signaling cascade. CANCER SCIENCE 103:1640-1650. 10.1111/j.1349-7006.2012.02367.x

Zeng, T., Chen, C., Yang, P., Zuo, W., Liu, X., and Zhang, Y. 2019. A Protective Role for RHOJ in NonSmall Cell Lung Cancer Based on Integrated Bioinformatics Analysis. JOURNAL OF COMPUTATIONAL BIOLOGY. 10.1089/cmb.2019.0209

Zhou, Y., Zhou, B., Pache, L., Chang, M., Khodabakhshi, A.H., Tanaseichuk, O., Benner, C., and Chanda, S.K. 2019. Metascape provides a biologist-oriented resource for the analysis of systems-level datasets. Nature Communications 10:1523. 10.1038/s41467-019-09234-6 


\section{Table $\mathbf{1}$ (on next page)}

Two hundred thirty-two differentially expressed genes (DEGs) were screened from three profile datasets. 
1

2 Table1. Two hundred thirty-two differentially expressed genes (DEGs) were screened from three profile 3

\begin{tabular}{|c|c|}
\hline DEGs & Genes symbol \\
\hline \multirow[t]{4}{*}{ Upregulated (40) } & G2E3,GDA ,EPT1 ,KPNA2, IGH ,ANLN ,BIRC5 ,HSPD1 ,PTK2 ,BC017398, MIR3934, SAPCD2, IGHD \\
\hline & , AGO2,GTSE1 ,CBX2 ,PTBP3 ,ADAM28, CCNB2, LRP8 ,NFE2L3,KRT16, IGHG1 ,NME1 ,COL12A1 ,EYA2 \\
\hline & ,LEPREL4 ,LRRC15,BCL2L11 ,ATAD2 ,MTA3 , HIST1H2BG ,PCDH19 ,SLC1A4,HELLS ,GBP6 ,FERMT1 ,KRT5 \\
\hline & ,HOXC6, CDKN2A. \\
\hline \multirow[t]{16}{*}{ Downregulated (192) } & PFKP ,ERG ,HIGD1B ,VGLL3 ,IPW ,MAOA,CDK1 ,RSRC1 ,SPTBN1 ,SNX25,UNC13B ,PPBP ,QKI ,SPG20 \\
\hline & , MAD2L1, SORBS2 ,CCM2L , WIF1 , GIMAP6 ,SOCS3 , MAGI1, LMO7, CCL23, PCDP1 ,OGN ,KRT4 ,SFTA3 \\
\hline & , CEACAM5 ,PDE5A ,SLC16A4 ,PDZD2 ,WISP1 ,HBB ,ITGAX ,TM9SF3 ,LPL ,COL11A1 ,ODF3B ,CASP4 \\
\hline & , ROR1 ,SYNM ,UGT8 ,FKBP11 ,SESTD1 ,SLC4A4 ,RP699M1.2 ,CTTN ,NEK2 ,SMAD6 ,MEF2C ,ERBB4 \\
\hline & , RP115C23.1 , MACF1 ,MAGEA10,MAGEA5, ITIH5 ,SIGLEC17P ,TBX5 ,PARVA ,PPAP2C ,AQP4 ,SLC47A1 \\
\hline & ,SERPINA1 ,COL4A3 ,IL1RL1 ,MCEMP1 ,CYP4V2 ,TRPV2 ,STOM ,KIAA1244 ,EDNRB ,ST3GAL6 ,SOX17 \\
\hline & ,TNS1 ,CAMK2N1 ,POLQ ,CACNA1D ,RGS5 ,PTGDS ,GAGE12B, EIF5 ,SERPINB1 ,PTPN21 ,CST6 ,STRBP \\
\hline & , NAV1 ,SHROOM3 ,CNTNAP2 ZNNF280B, LMO3, MBIP, IL33, ARHGAP6,RNF125,CYP2B6 ,ANK3 ,DAPL1 \\
\hline & , KCTD1 ,TACC2 ,MITF ,LILRB2 ,HOXA1 ,CSF3R ,LOC643733, HNMT,GNAS ,SLC27A3 ,SERPINB9 ,C3 \\
\hline & ,SERPING1 ,AFAP1L1 ,SULT1A2 , ZFPM2 ,SEC63, ADRB1 ,SVEP1 ,FYN,COL5A2 ,LOC101928198 ,PLAC9 \\
\hline & , MSR1, LST1 ,DOCK4 ,FRMD4A , KLF9, PDK4 ,EMCN ,TSPAN12, ,CA4, SRPX2 ,SIRPA ,APOH ,CLEC7A \\
\hline & , NCKAP1L ,LHFP , GLS ,CFLAR ,ACACB , RUFY3 ,SOBP ,PMP22 ,P2RX7, LEPREL1 ,LPCAT1 ,SOX2 ,IQGAP2 \\
\hline & , OTUD1, FRMD3, DOCK2 , BTNL9, UCHL1 ,CLEC2B ,TBX2 ,TMEM237, PPARG ,HLADQB1 ,LMOD1 \\
\hline & ,SUGT1 ,LRRN4 ,RGCC ,ADRB2 ,CMAHP ,SEMA6A ,HMGA2 ,CCDC68 ,SREK1IP1 ,MYLIP , DOCK9 \\
\hline & , MARCKS , RORA ,SORBS1, GUCY1A2 ,STXBP6 ,STX12 ,CASC5 ,CALML3 ,CKAP2 ,ICAM1 ,FGF18, ZEB2 \\
\hline & , DSG3 ,LGI3, TTN ,AKAP13, SLC34A2 ,STAC ,TAPT1 ,SEMA5A ,SYNPO,CAST ,SLCO4C1 ,HBA1 \\
\hline
\end{tabular}

4 
Table 2 (on next page)

Pathway and Process Enrichment Analysis 
Table 2. Pathway and Process Enrichment Analysis

\begin{tabular}{|c|c|c|c|c|c|}
\hline GO & Category & Description & Count & $\%$ & $\log 10(P)$ \\
\hline \multicolumn{6}{|l|}{ Upregulated } \\
\hline M236 & Canonical Pathways & PID DELTA NP63 PATHWAY & 4 & 10.53 & -6.05 \\
\hline M176 & Canonical Pathways & PID FOXM1 PATHWAY & 3 & 7.89 & -4.47 \\
\hline R-HSA-5693606 & Reactome Gene sets & DNA Double Strand Break Response & 3 & 7.89 & -3.62 \\
\hline M66 & Canonical Pathways & PID MYC ACTIV PATHWAY & 4 & 7.89 & -3.58 \\
\hline G0:0007160 & GO Biological Processes & Ell-matrix adhesion & 5 & 10.53 & -3.37 \\
\hline GO:1903828 & GO Biological Processes & $\begin{array}{l}\text { Negative regulation of cellular protein } \\
\text { localization }\end{array}$ & 3 & 7.89 & -3.16 \\
\hline G0:0008637 & GO Biological Processes & Apoptotic mitochondrial changes & 3 & 7.89 & -3.01 \\
\hline R-HSA-9006925 & Reactome Gene sets & $\begin{array}{l}\text { Intracellular signaling by second } \\
\text { messenger }\end{array}$ & 3 & 10.53 & -2.89 \\
\hline G0:0042742 & GO Biological Processes & Defense response to bacterium & 4 & 10.53 & -2.78 \\
\hline G0:0051301 & GO Biological Processes & Cell division & 5 & 13.16 & -2.65 \\
\hline GO:0017038 & GO Biological Processes & Protein import & 3 & 7.89 & -2.48 \\
\hline G0:0048872 & GO Biological Processes & Homeostasis of number of cells & 3 & 7.89 & -2.15 \\
\hline \multicolumn{6}{|l|}{ Downregulated } \\
\hline G0:0048589 & GO Biological Processes & Developmental growth & 23 & 12.17 & -8.59 \\
\hline G0: 0030036 & GO Biological Processes & Actin cytoskeleton organization & 22 & 11.64 & -8.04 \\
\hline GO:0001568 & GO Biological Processes & Blood vessel development & 22 & 11.64 & -6.79 \\
\hline GO:0002274 & GO Biological Processes & Myeloid leukocyte activation & 19 & 10.05 & -6.01 \\
\hline GO:0052548 & GO Biological Processes & Regulation of endopeptidase activity & 15 & 7.94 & -5.93 \\
\hline G0:0060485 & GO Biological Processes & Mesenchyme development & 12 & 6.35 & -5.70 \\
\hline R-HSA-109582 & Reactome Gene sets & Hemostasis & 18 & 9.52 & -5.67 \\
\hline G0:0010927 & GO Biological Processes & $\begin{array}{l}\text { Cellular component assembly involved in } \\
\text { morphogenesis }\end{array}$ & 8 & 4.23 & -5.60 \\
\hline G0:0003012 & GO Biological Processes & Muscle system process & 15 & 7.94 & -5.36 \\
\hline G0:1905114 & GO Biological Processes & $\begin{array}{l}\text { Cell surface receptor signaling pathway } \\
\text { involved in cell-cell signaling }\end{array}$ & 17 & 8.99 & -5.08 \\
\hline G0:0008285 & GO Biological Processes & Negative regulation of cell proliferation & 19 & 10.05 & -5.00 \\
\hline hsa04924 & KEGG Pathway & Renin secretion & 6 & 3.17 & -4.92 \\
\hline GO: 0048588 & GO Biological Processes & Developmental cell growth & 10 & 5.29 & -4.86 \\
\hline
\end{tabular}




\begin{tabular}{|c|c|c|c|c|c|}
\hline G0:0010884 & GO Biological Processes & Positive regulation of lipid storage & 4 & 2.12 & -4.62 \\
\hline G0:0003013 & GO Biological Processes & Circulatory system process & 15 & 7.94 & -4.55 \\
\hline G0: 0051345 & Reactome Gene Sets & Posithive regulation of hydrolase activity & 18 & 9.52 & -4.49 \\
\hline G0: 0043408 & GO Biological Processes & Regulation of MAPK cascade & 18 & 9.52 & -4.44 \\
\hline R-HSA-1247673 & GO Biological Processes & Erythrocytes take up oxygen release & 3 & 1.59 & -4.42 \\
\hline GO:0099612 & GO Biological Processes & Protein localization to axon & 3 & 1.59 & -4.42 \\
\hline GO: 0045444 & GO Biological Processes & Fat cell differentiation & 9 & 4.76 & -4.21 \\
\hline
\end{tabular}


Table 3 (on next page)

Functional roles of 10 hub genes. 
1 Table 3. Functional roles of 10 hub genes

\begin{tabular}{|c|c|c|}
\hline Genes symbol & Full name & Function \\
\hline MAD2L1 & Mitotic arrest deficient 2 like 1 & preventing the onset of anaphase \\
\hline POLQ & DNA polymerase $\theta$ & alternative nonhomologous end joining \\
\hline HELLS & Helicase, lymphoid specific & DNA strand separation \\
\hline ANLN & Aniline actin binding protein & cell growth and migration \\
\hline BIRC5 & Bucovina IAP repeat containing 5 & preventing apoptotic cell death \\
\hline ATAD2 & $\begin{array}{c}\text { ATPase family AAA domain } \\
\text { containing2 }\end{array}$ & chaperone-like functions \\
\hline CCNB2 & Cyclin B2 & the cell cycle regulatory machinery \\
\hline PTK2 & Protein tyrosine kinase 2 & cytoplasmic protein tyrosine kinase \\
\hline ICAM1 & Intercellular adhesion molecule1 & endothelial cells and cells of the immune system \\
\hline ITGAX & Integrin subunit alpha $X$ & encoding the integrin alpha $X$ chain protein \\
\hline
\end{tabular}


Figure 1

Fig. 1 Functional and pathway enrichment analysis of DEGs.

(A) GO terms and KEGG pathway were presented, and each band represents one enriched term or pathway colored according to the $-\log 10 \mathrm{P}$ value. (B) Network of the enriched terms and pathways. Nodes represent enriched terms or pathways with node size indicating the number of DEGs involved in. Nodes sharing the same cluster are typically close to each other, and the thicker the edge displayed, the higher the similarity is represented.

A

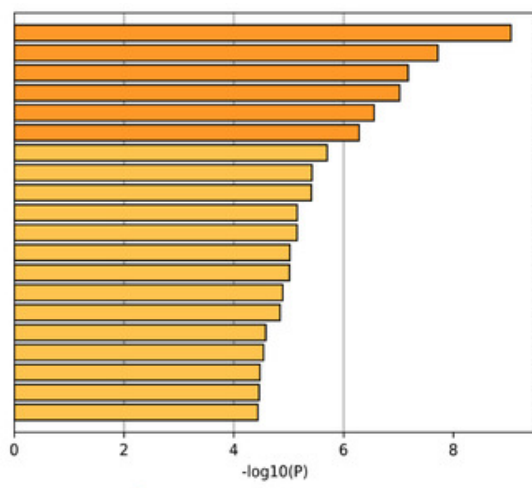

GO:0048589: developmental growth G0:0097435: Supramolecular fiber organization

60:0001667: ameboidal-type cell migration GO:0001568: blood vessel development GO:0052548: regulation of endopeptidase activity R-HSA-109582: Hemostasis

0.0008285: negative regulation of cell proliferation

G0:0051222: positive regulation of protein transport

G0:1905114: cell suffece receptor signaling pathway inuolved in cell cell signaling

60:0003012: muscle system process

GO:0010927: cellular component assembly involved in morphogenesis

GO:0060485: mesenchyme development

GO:0002274: myeloid leukocyte activation

0:0050954: sensory perception of mech

60:0030155: regulation of cell adhesion

hsa04924: Renin secretion

Go.0007044: cell-substrate junction assembly 60:0048598: embryonic morphogenesis

B
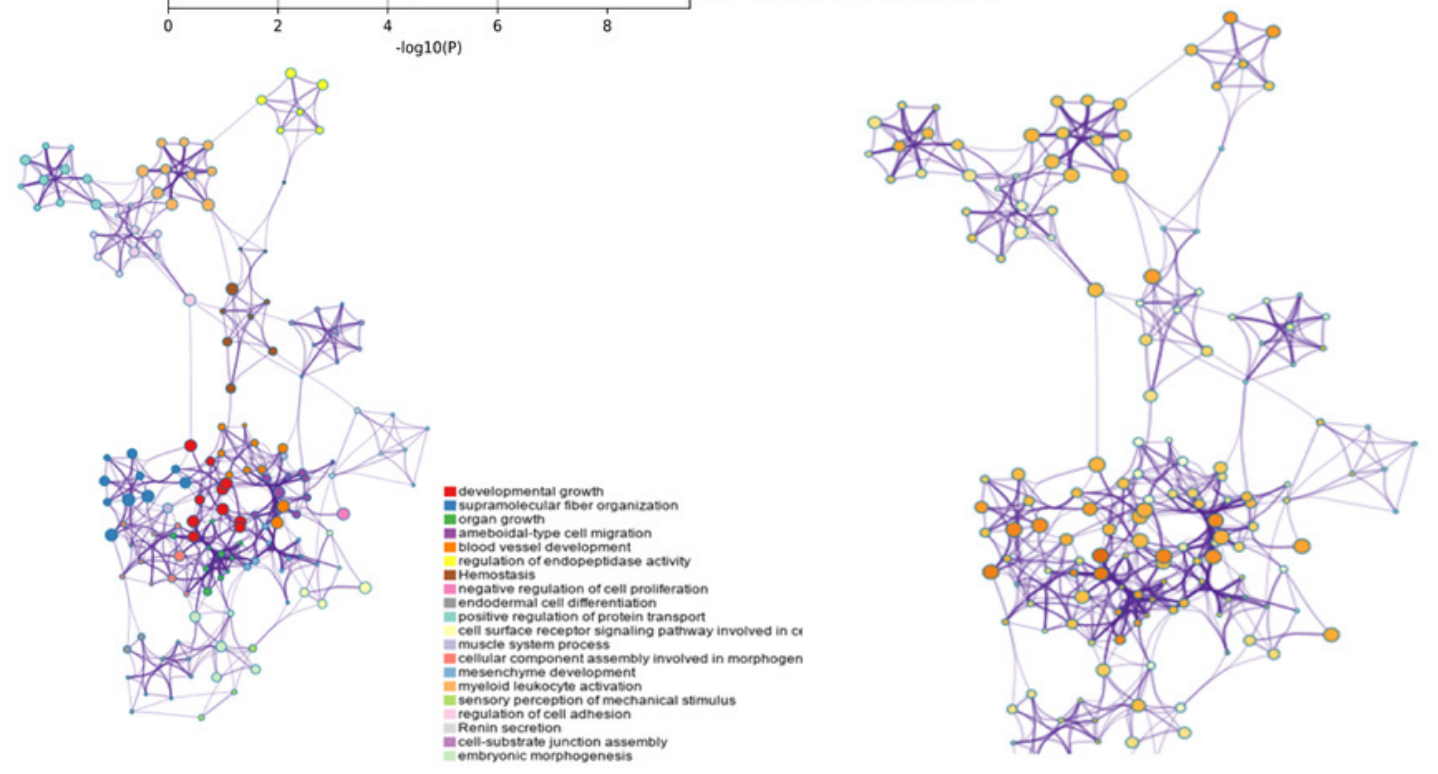

$10^{-2}$

$10^{-3}$

$10^{-4}$

$10^{-6}$

$10^{-10}$

$10^{-20}$ 
Figure 2

Fig. 2 The distribution of differential genes between GSE18842, GSE19188 and GSE27262.

(A) DEGs were selected with $p<0.05$ and [log FC] $>1$ among the mRNA expression profiling sets. (B-C) The PPI network of DEGs was constructed using Cytoscape (upregulated genes are marked in light red; downregulated genes are marked in light blue).

A

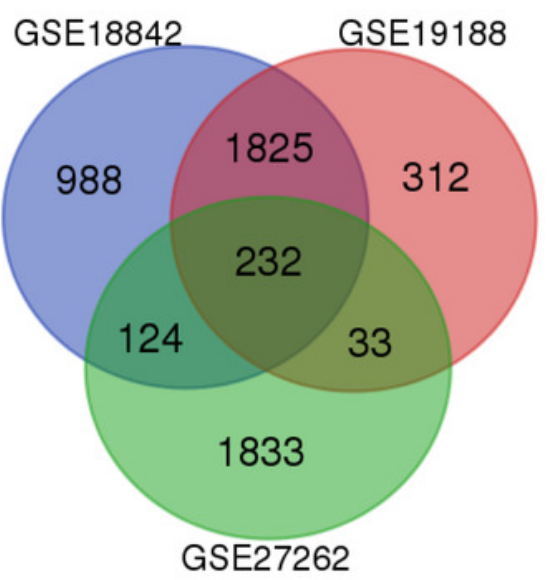

$\mathrm{C}$

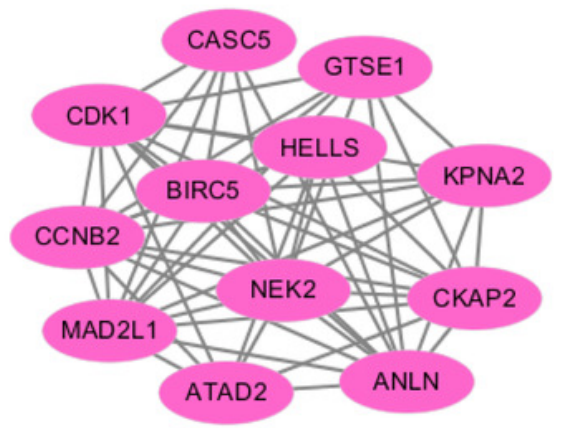

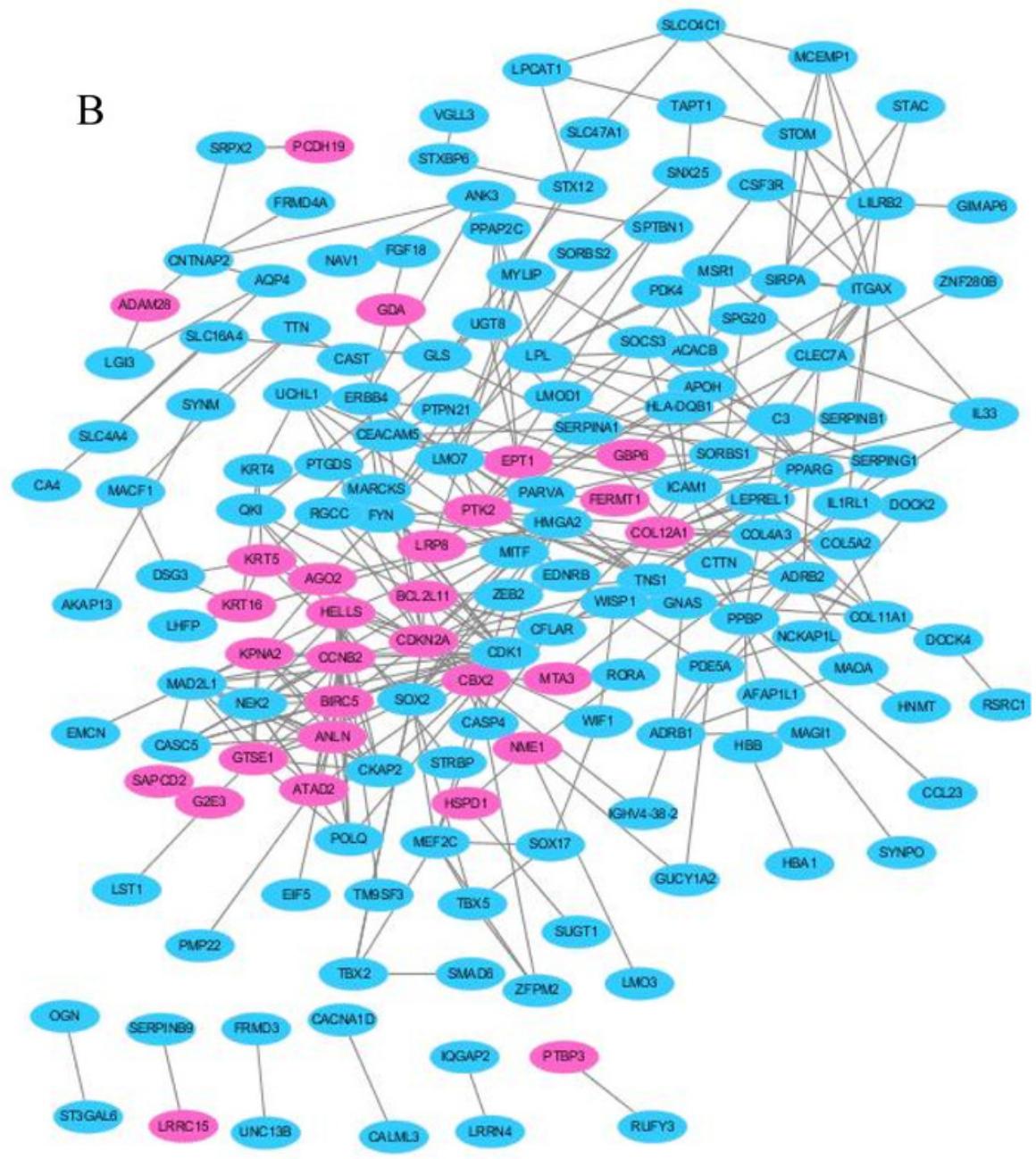


Figure 3

Fig. 3 Functional and pathway enrichment analysis of the PPI module.

(A). GO terms and KEGG pathway were presented, and each band represents one enriched term or pathway colored according to the $-\log 10 \mathrm{P}$ value. (B). Network of the enriched terms and pathways. Nodes represent enriched terms or pathways with node size indicating the number of DEGs involved in.

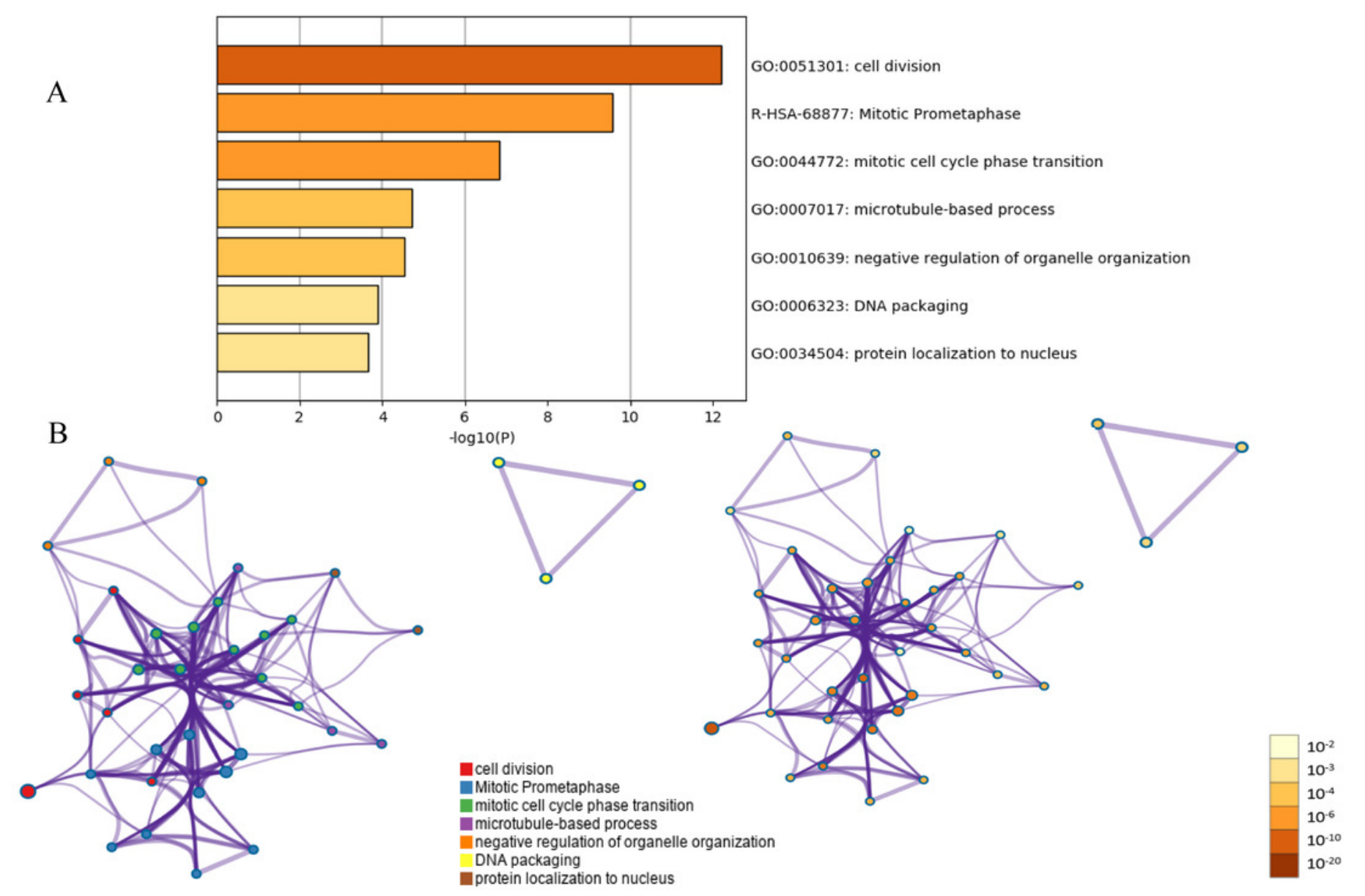




\section{Figure 4}

Hub gene selection and analysis

(A)Hub genes and their co-expression genes were analyzed using cBioPortal. Nodes with bold black outline represent hub genes. Nodes with thin black outline represent the co-expression genes.(B)Hierarchical clustering of hub genes was constructed using UCSC. (C)The biological process analysis of hub genes was constructed using BiNGO. 
B
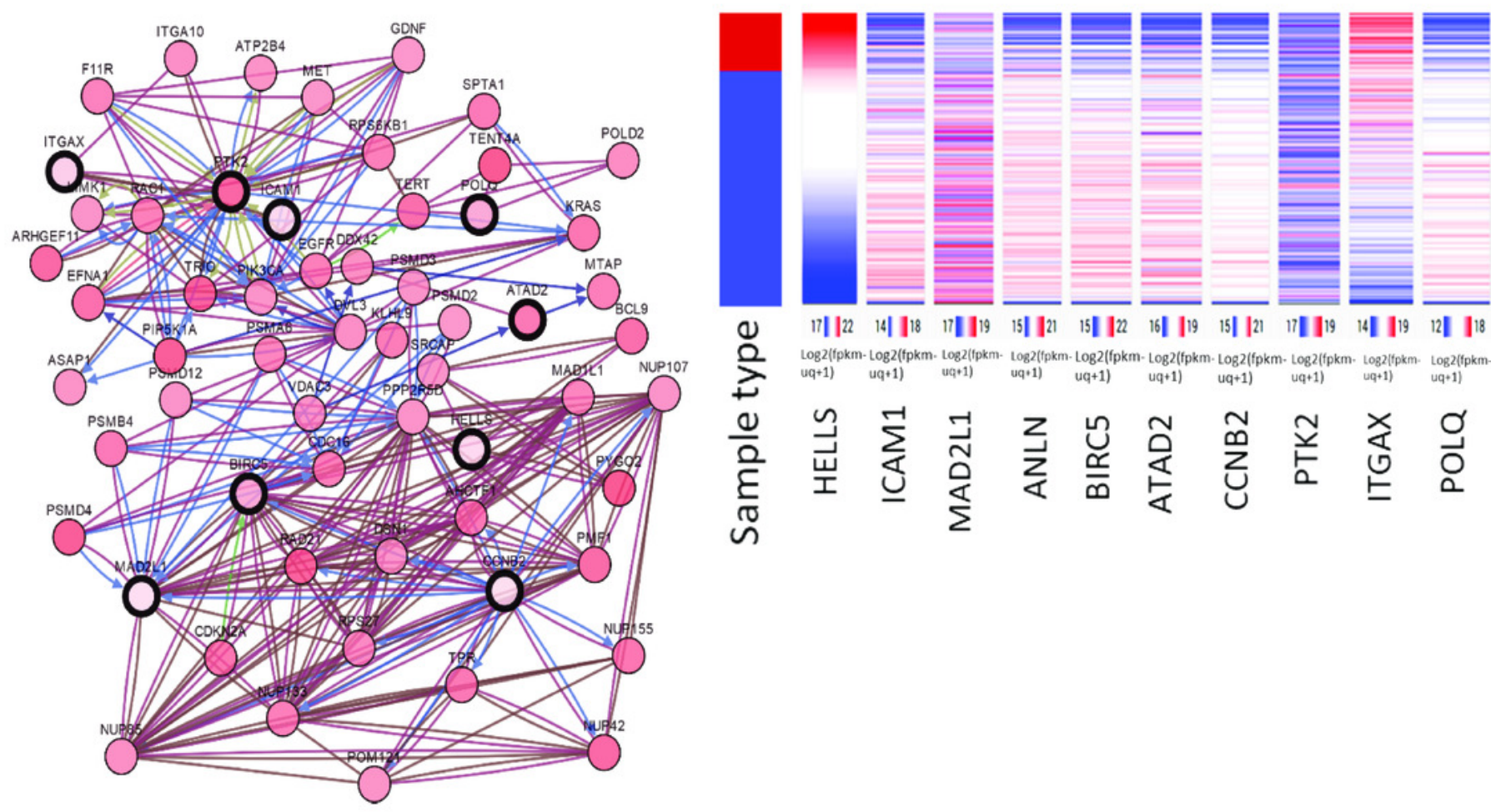

C

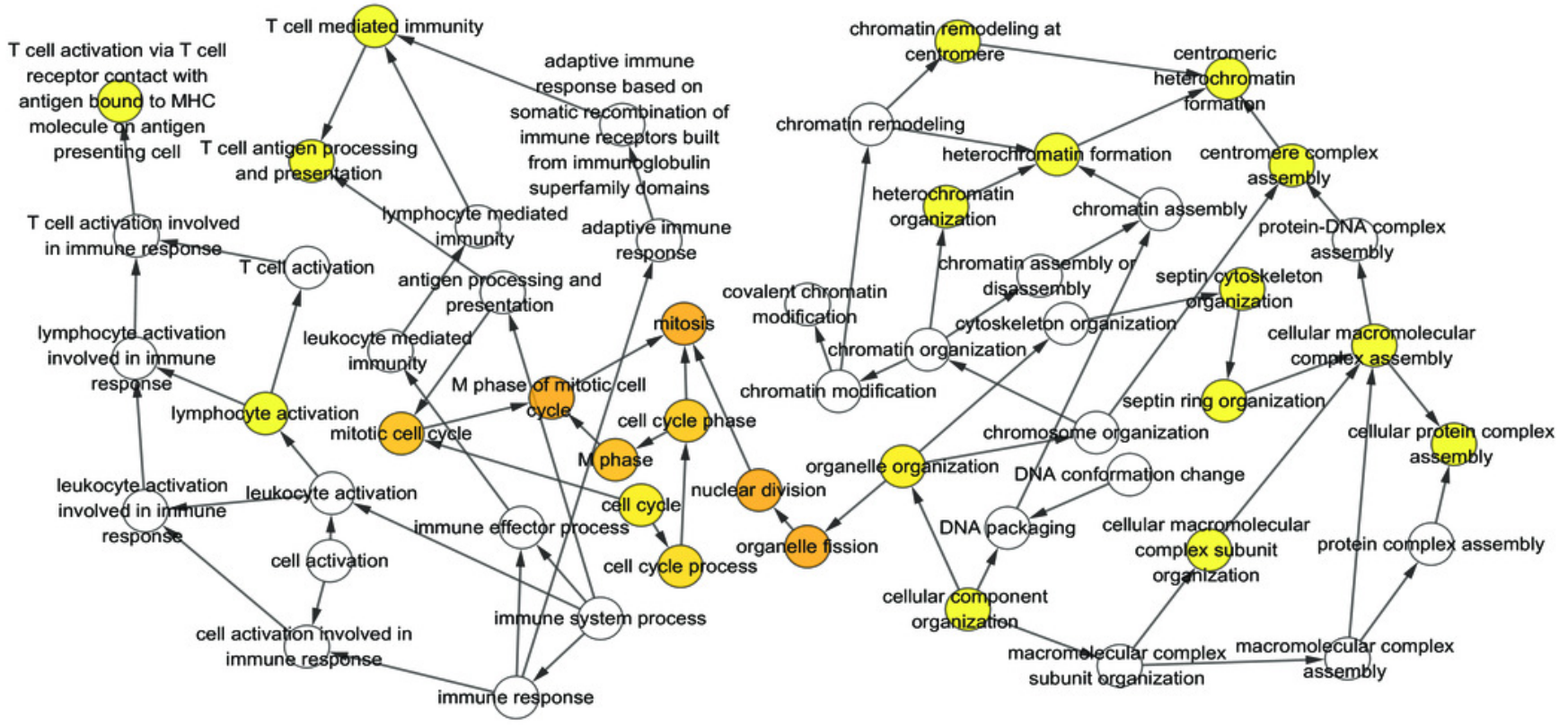


Figure 5

Overall survival and disease-free survival analyses of different HELLS expression lung cancer patients.

(A-F) Overall survival and disease-free survival analyses of the HELLS were performed in TCGA online website. $]$ G-H $[$ The mRNA level of HELLS was evaluated in lung cancer using GEPIA analysis, $P<0.05$ was considered to indicate a statistically significant difference. 

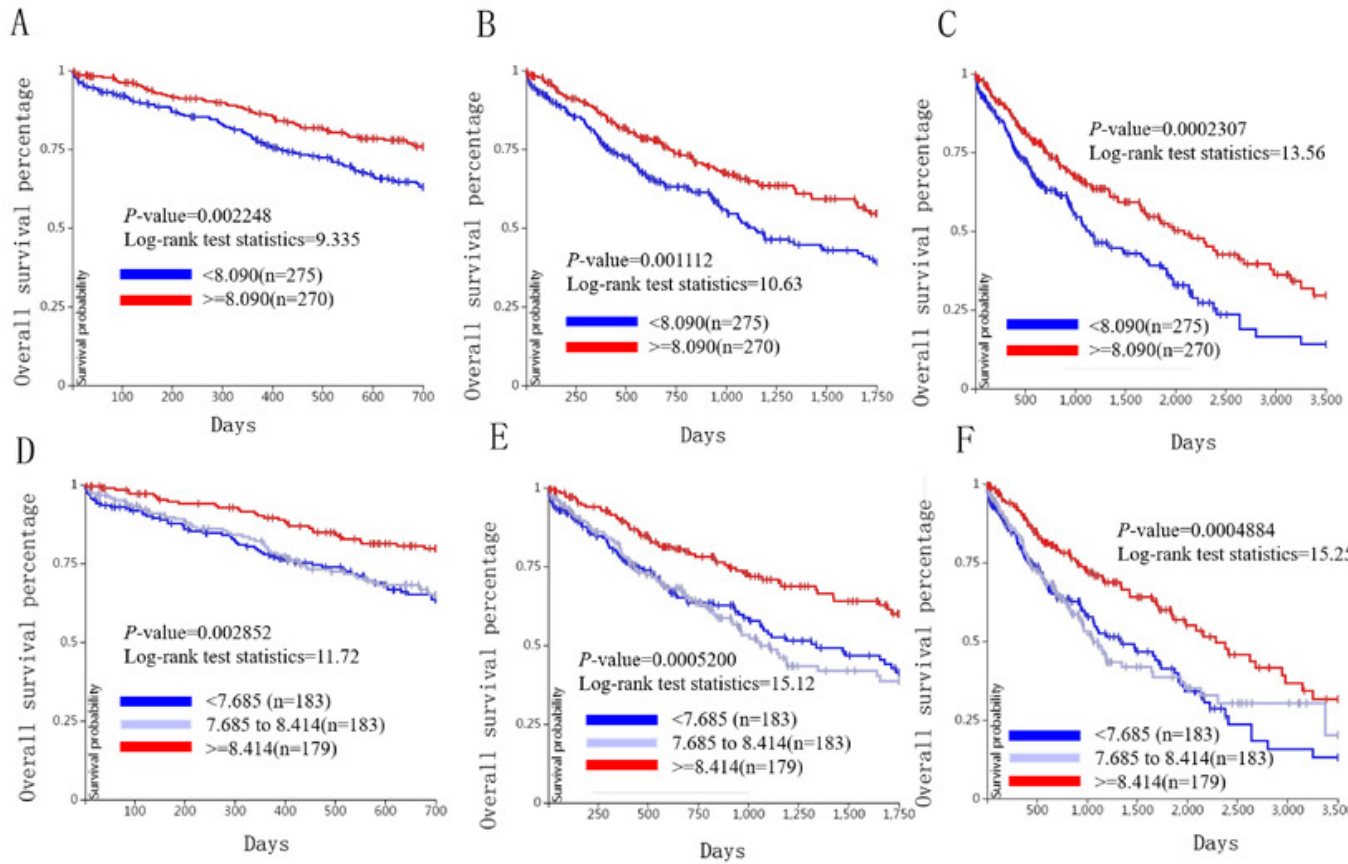

E
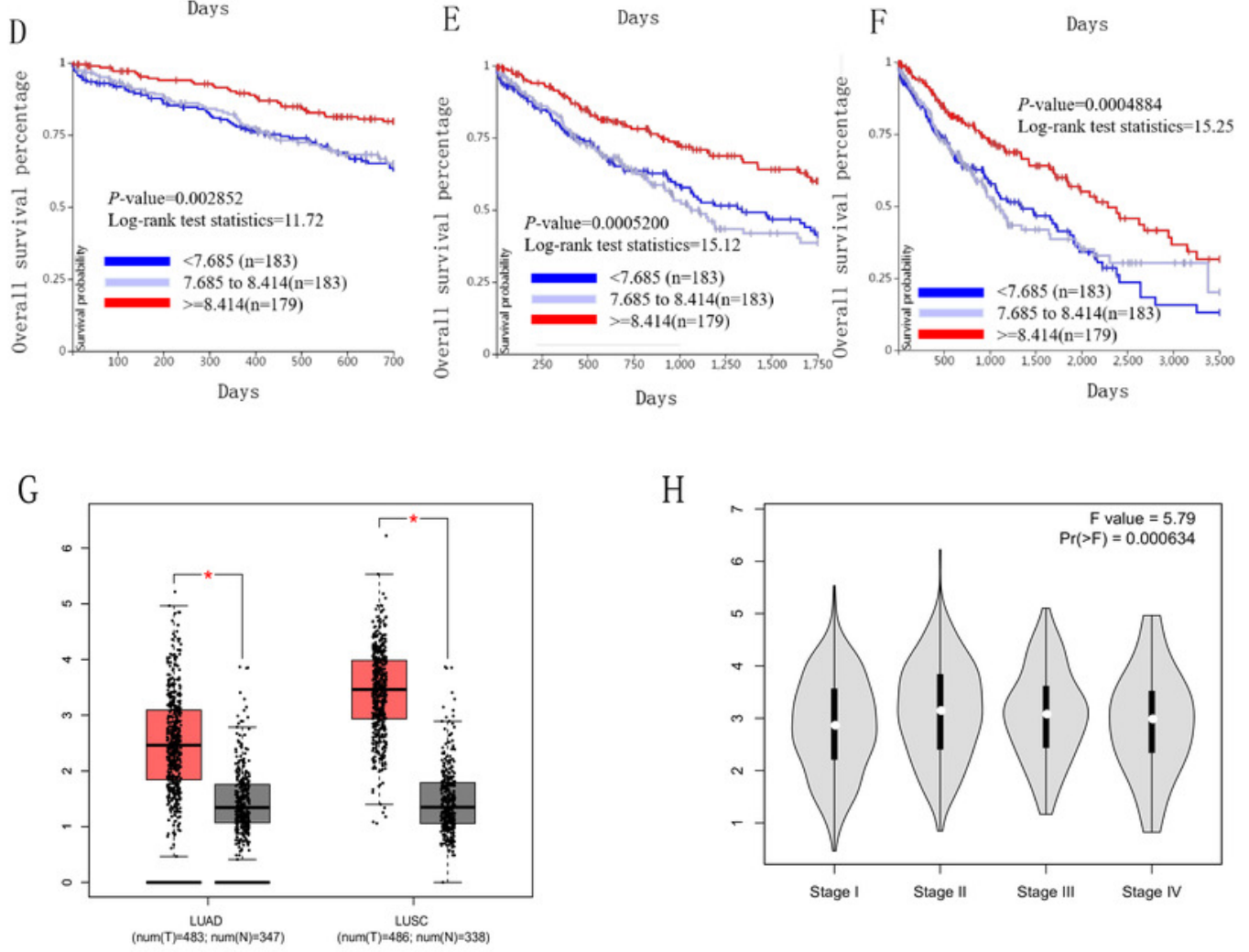
Figure 6

Overall survival and disease-free survival analyses of different ICAM1 expression lung cancer patients.

(A-F) Overall survival and disease-free survival analyses of the ICAM1 were performed in TCGA online website. (G-H) The mRNA level of ICAM1 was evaluated in lung cancer using GEPIA analysis $\mathrm{P}<0.05$ was considered to indicate a statistically significant difference. 
A

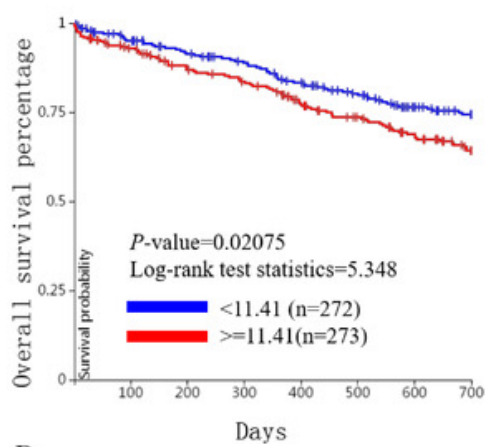

D

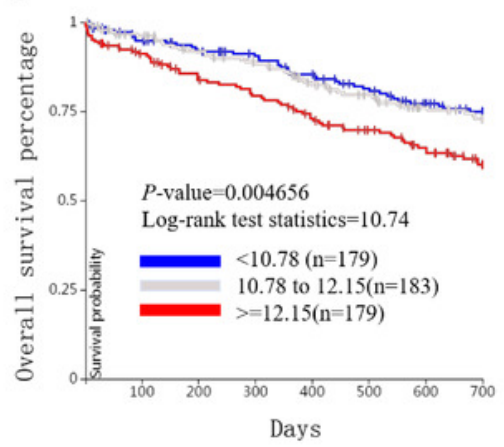

B
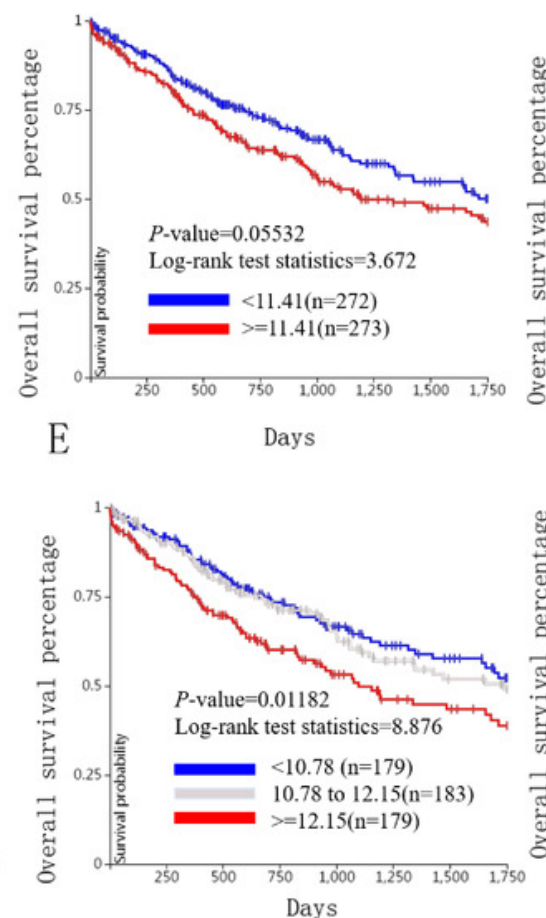

C
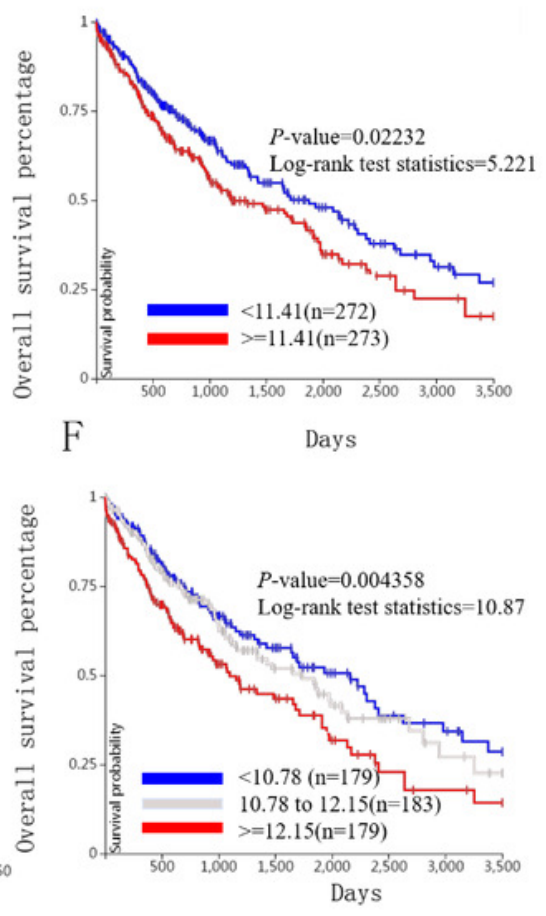

G

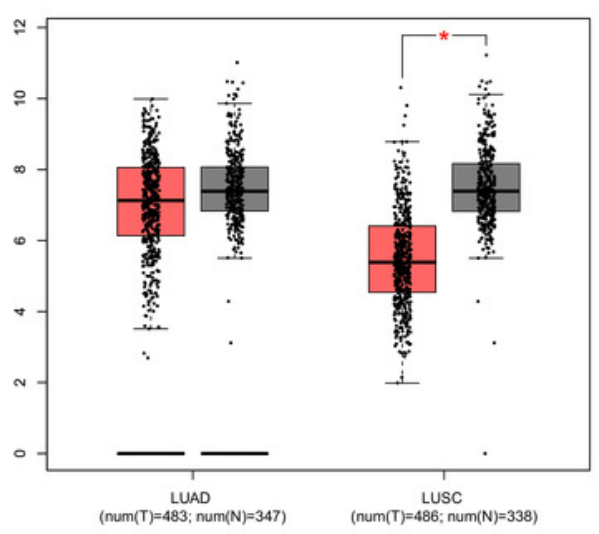

$\mathrm{H}$

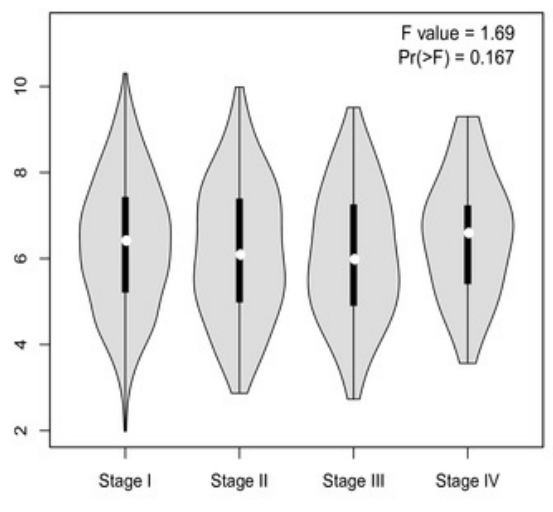




\section{Figure 7}

The mRNA level of HELLS and ICAM1, the PPI network of HELLS and ICAM1 were constructed.

(A-B) The mRNA level of HELLS and ICAM1 were evaluated in lung cancer among four studies using ONCOMINE analysis. (C-D) PPI network of HELLS and ICAM1 were constructed by STRING database. 
A

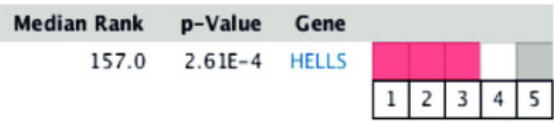

\section{Legend}

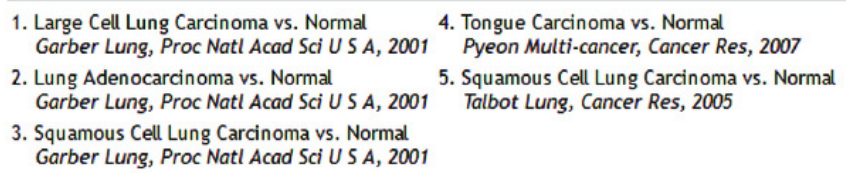

$\begin{array}{llllllll}1 & 5 & 10 & 25 & 25 & 10 & 5 & 1\end{array}$

$\square \square \square \square \square \square \square \square \square \square$ Not measured

The rank for a gene is the median rank for that gene across each of the analyses.
The p-Value for a gene is its p-Value for the median-ranked analysis.

B

Comparison of ICAM1 Across 5 Analyses

Over-expression

Median Rank p-Value Gene

$3213.5 \quad 0.005 \quad$ ICAMI

\begin{tabular}{|l|l|l|l|l|}
\hline 1 & 2 & 3 & 4 & 5 \\
\hline
\end{tabular}

Legend

1. Large Cell Lung Carcinoma vs. Normal 4. Tongue Carcinoma vs. Normal

Garber Lung, Proc Natl Acad Sci U S A, 2001 Pyeon Multi-cancer, Cancer Res, 2007

2. Lung Adenocarcinoma vs. Normal $\quad$ 5. Squamous Cell Lung Carcinoma vs. Normal Garber Lung, Proc Natl Acad Sci U S A, 2001 Talbot Lung, Cancer Res, 2005

3. Squamous Cell Lung Carcinoma vs. Normal Garber Lung, Proc Natl Acad Sci U S A, 2001

\section{$\begin{array}{llllllll}1 & 5 & 10 & 25 & 25 & 10 & 5 & 1\end{array}$}

\section{$\square \square \square \square \square \square \square \square \square \square$ Not measured}

The rank for a gene is the median rank for that gene across each of the analyses.
The p-Value for a gene is its $\mathrm{p}$-Value for the median-ranked analysis.

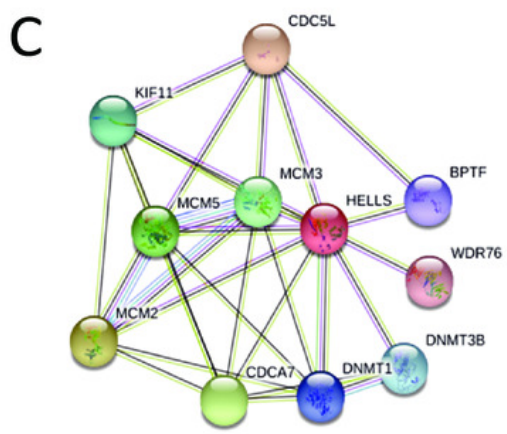

Node Color

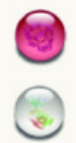

colored nodes:

query proteins and first shell of interactors

white nodes:

second shell of interactors

Known Interactions

$\ominus-\ominus$ from curated databases

$\odot-\odot$ experimentally determined

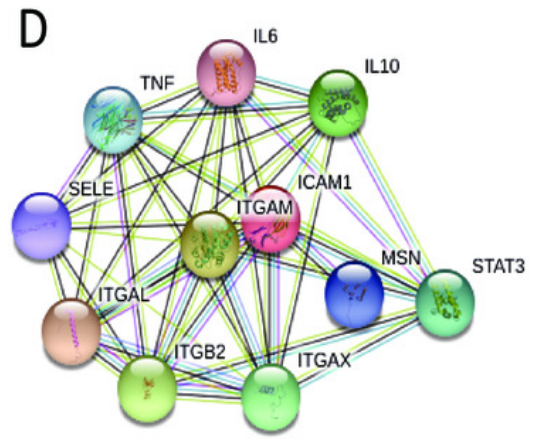

Node Content

empty nodes

proteins of unknown $3 D$ structure

filled nodes:

some $3 D$ structure is known or predicted
Predicted Interactions

○- - gene neighborhood

$\mathrm{C}-\mathrm{S}$ gene fusions

○-○ gene co-occurrence
Others

e- - textmining

C-O co-expression

○-O protein homology 
Figure 8

Fig. 8 The expression levels of ICAM1 and HELLS in the lung cancer samples and the lung cancer cells.

$(A, B)$ The expression levels of ICAM1 and HELLS in the lung cancer samples. $(C, D)$ The expression levels of ICAM1 and HELLS in the lung cancer cells.
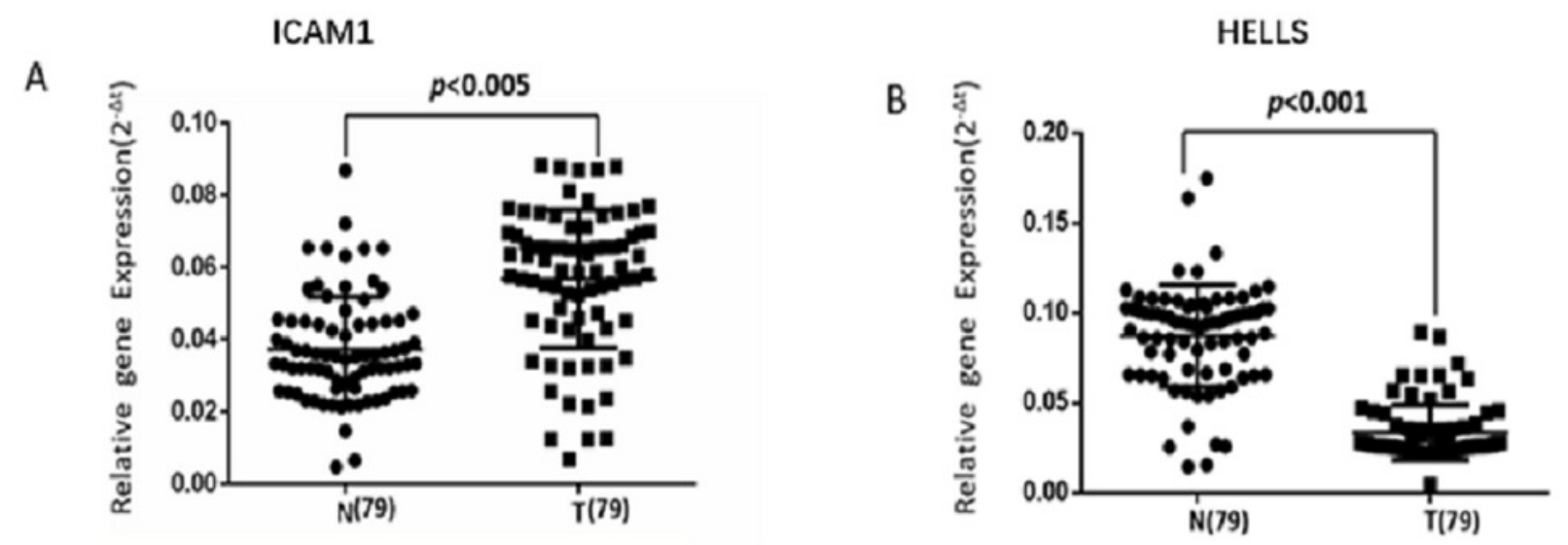

c

ICAM1 quantitative expression
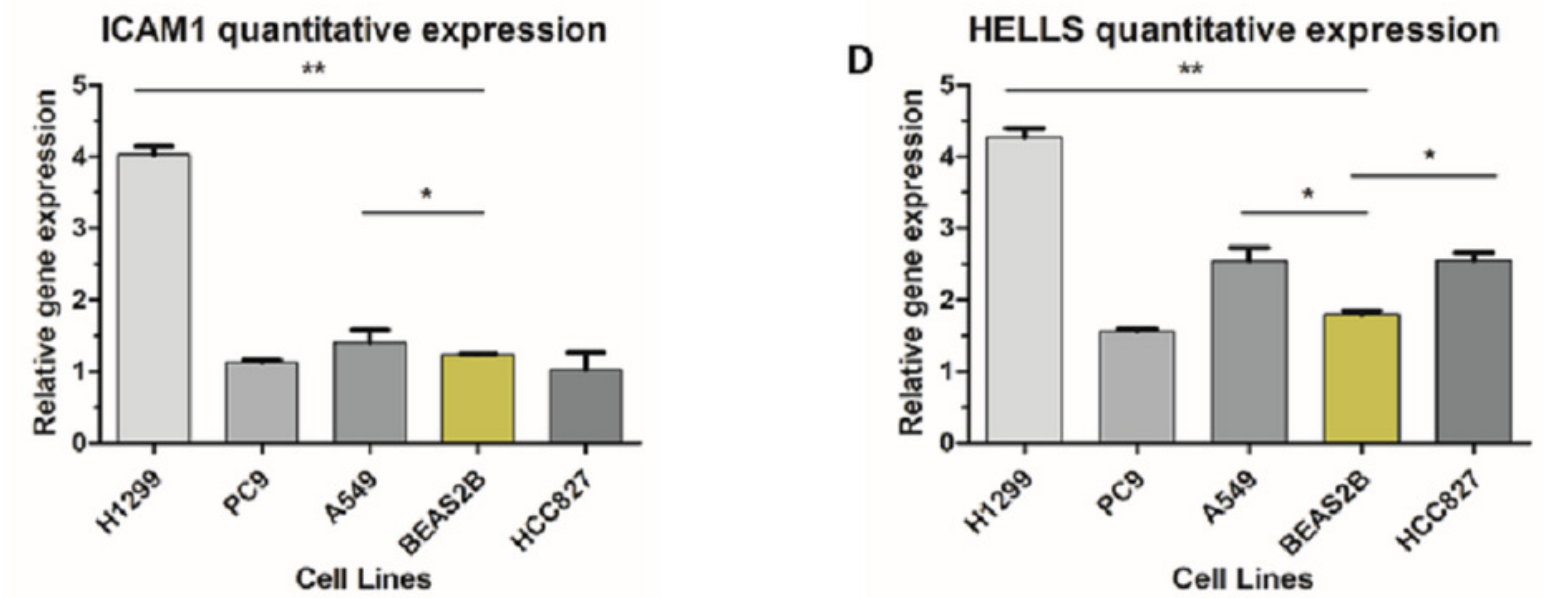